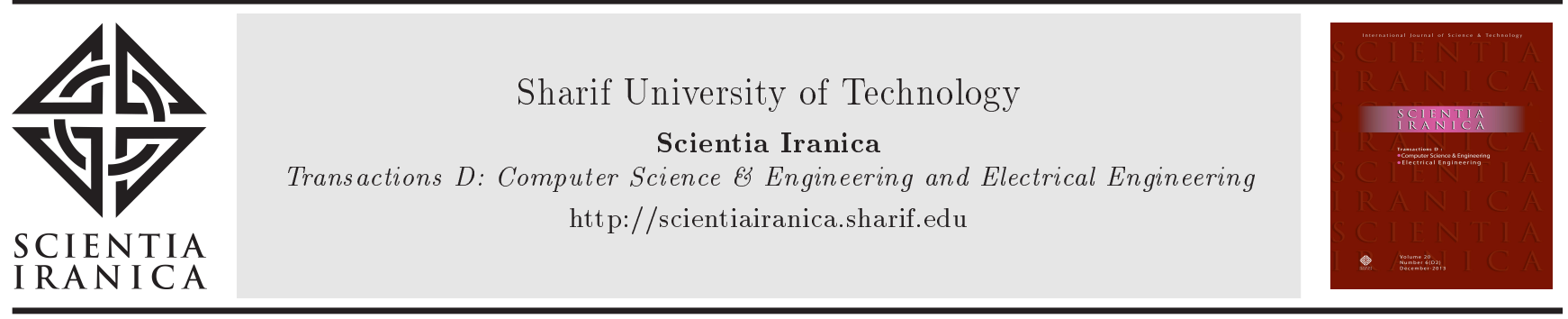

Research Note

\title{
A design of mass-spring type piezoelectric energy harvesting
}

\author{
S. Akkaya Oy* \\ Faculty of Fatsa Marine Sciences, Ordu University, Ordu, 52400, Turkey.
}

Received 16 October 2019; received in revised form 18 January 2020; accepted 27 April 2020

\section{KEYWORDS}

Wind;

Piezoelectric

transducer;

Energy harvester;

Spring;

Low power generator.

\begin{abstract}
This study introduces an experimental power generation system based on piezoelectric energy conversion for low-power applications such as wireless sensor nodes. Piezoelectric Transducers (PZTs) are especially suitable for energy harvesting from the ambiance. A PZT is able to produce an open-circuit voltage at a hundred-volt level. However, the acquired current may be achieved on a nano-micro scale. Therefore, PZT Energy Harvesters (PEH) have a very limited power generation capability. In order to generate greater output power, a series of PZTs should be applied to a PEH with a proper electrical connection. In this paper, PEH is presented with a novel circuit topology to increase the output power. The experimental system uses 20 diaphragm type PZTs with a spring of $15 \mathrm{~cm}$ mounted at the center of each transducer. The required vibration is produced using a spring-mass structure of each PZT. Mass-spring structure vibrates by the wind effect. The maximum output power of the presented experimental generator at a nominal wind speed of $12 \mathrm{~m} / \mathrm{s}$ is around $10.8 \mathrm{~mJ}$. The obtained results indicate that the proposed experimental generator is suitable for low-power applications, and the output power of the generator can be increased by using more PTZs connected via circuit topology.
\end{abstract}

(C) 2021 Sharif University of Technology. All rights reserved.

\section{Introduction}

Energy harvesting technologies have gained significance in recent years as a result of recycling dissipated energy in several environments such as wind, light, heat, and vibration [1]. Owing to its high efficiency, simple structure, high voltage generation capability, and low cost, piezoelectric energy harvesting plays a key role in these technologies $[2,3]$. By using piezoelectric material, vibration energy in the environment can be converted to electrical energy [4].

There are many studies on piezoelectric energy

*. Tel.: +90-452-423-5053

E-mail address: sibelakkayaoy@gmail.com

doi: $10.24200 /$ sci.2020.54665.3856 harvesting in the literature that may be considered in three categories based on the vibration source used, interface circuit used, and amount of energy produced. For example, Wei and Taghavifar [5] developed an energy harvester based on the harmonic movements of a vehicle suspension system. When tested on gravel and straight roads, this harvester produced an output power of $57.87 \mathrm{~W}$ at a speed of $13 \mathrm{~km} / \mathrm{h}$. The average power was found to increase in direct proportion with the road amplitude. Khoshnoud et al. [6] also developed an energy harvesting system on a vehicle suspension system that employed Regenerative Force Actuator (RFA) to utilize vibration energy, thus achieving a maximum power of $984.4 \mathrm{~W}$ at a frequency of $20 \mathrm{~Hz}$. Ozaki and Hamaguchi [7] designed and fabricated a bioinspired flapping-wing micro aerial vehicle with piezoelectric direct-driven actuation. The total 
mass of the prototype was $598 \mathrm{mg}$, and the maximum measured lift force was $6.52 \mathrm{mN}$ at a driving voltage of $100 \mathrm{~V}$.

Wave energy is another source of vibration for piezoelectric transducers. Xie et al. [8] developed an ocean wave energy harvester that generated electricity by collecting the effects of the transverse wave motion of water particles on a piezoelectric plate. From simulation, this harvester could generate energy up to $30 \mathrm{~W}$. Song et al. [9] developed an upright vortexinduced piezoelectric energy harvester from which they obtained an output power of $84.49 \mu \mathrm{W}$ at the energy density of $60.35 \mathrm{~mW} / \mathrm{m}^{2}$ and resonance vibration rate of $0.35 \mathrm{~m} / \mathrm{s}$. Shan et al. [10] developed a Macro Fiber Composite (MFC) Piezoelectric Energy Harvester $(\mathrm{PEH})$. In their experiments, they produced a maximum output power of $1.32 \mu \mathrm{W}$ with the power density of $1.1 \mathrm{~mW} / \mathrm{m}^{2}$ at a water velocity of $0.5 \mathrm{~m} / \mathrm{s}$. Yeong-min et al. [11] developed a piezoelectric energyharvesting device based on constant wave motion. The average current and voltage were $71.4 \mu \mathrm{A}$ and $2.42 \mathrm{~V}$ with the output power of $0.173 \mathrm{~mW}$. Gianluca et al. [12] presented an energy harvester using the vibration effect of precipitation energy on the piezoelectric transducer and achieved an output power of $2.916 \times 10^{-8} \mathrm{~W}$.

The mechanical energy produced by the human body can also be converted into electrical energy by a piezoelectric transducer. For example, Wang et al. [13] investigated the optimum load resistance of a nonlinear energy harvester for real human motion and generated a maximum output power of $30.55 \mathrm{~mW}$ at a running speed of $7 \mathrm{~km} / \mathrm{h}$. Türkmen and Çelik [14] integrated energy harvesting using piezoelectric material in a shoe and demonstrated that a person weighing $90 \mathrm{~kg}$ could produce $1.43 \mathrm{~mW}$ of power during walking.

Flow-induced vibration is often used as an energy source for piezoelectric energy harvesters. Amini et al. [15] proposed a piezoelectric vertical beam with an attached end cylinder as an energy harvester in lowspeed flows. Piezoelectric energy harvesters based on vortex-induced vibration and galloping [16-19] have been extensively studied in the past several years. Shan et al. [20] and Song et al. [21] evaluated the performance of two tandem PEHs in water flow and found that the energy harvesting performance of the downstream PEH was enhanced due to the stimulation from the upstream $\mathrm{PEH}$ in the wake state. Zhang et al. [22] numerically investigated a $\mathrm{PEH}$ with the vortexinduced vibration. McCarthy et. al. [23] proposed forming a response from a fluttering PEH developed in smooth flow and in terms of replicated atmospheric boundary layer turbulence. Zou et al. [24] established a novel dual serial vortex-induced vibration energy harvesting system for enhancing energy harvesting.

Another common source of vibration for a piezoelectric harvester is wind. To be specific, Johar et al. [25] developed a nano-generator based on air-flow and vibration energy harvesting with the peak output voltage of $30 \mathrm{~V}$ and current of $1.43 \mu \mathrm{A}$. Ju et al. [26] studied an indirect impact-based piezoelectric vibration energy harvester using a Macro Fiber Composite beam as a piezoelectric cantilever that produced a maximum peak-to-peak open-circuit voltage of $42.2 \mathrm{~V}$ and average power of $633.7 \mu \mathrm{W}$. Sang et al. [27] designed a vibration-based hybrid energy harvester with the maximum output power of $10.7 \mathrm{~mW}$.

Akkaya and Özdemir [28] developed a wind-based generator that created vibrations for the piezoelectric generator by using the rotation of the unbalanced propeller system. The experimental results revealed that at a wind speed of $4.5-5 \mathrm{~m} / \mathrm{s}$ and load resistance of $150 \mathrm{~K} \Omega$, the maximum output power was $519 \mu \mathrm{W}$. Xie et al. [29] proposed a mathematical model of a cylindrical piezoelectric energy collector made of composite structures including a stator and a roller that converted mechanical energy into electrical energy using ambient vibration. According to the numerical simulation results, a cylinder energy harvester with a radius of $0.5 \mathrm{~m}$ and a rotating speed of $1 \mathrm{r} / \mathrm{s}$ would produce $60.13 \mathrm{~kW}$. Son et al. [30] developed a microscale vibration energy harvester with the maximum output power of $23.3 \mathrm{nW}$.

In this study, a new energy harvester system was designed to convert the mechanical energy provided by the vibration obtained from wind energy into electrical energy by means of piezoelectric converters. In this proposed system, a spring is mounted at the center of each piezo-sensor used in the circuit. These springs, which are subject to the effect of wind, provide the vibrations that piezo transducers require. At the same time, these springs prolong the vibration time and enable the mechanical energy to be used for a longer time.

Another important field of study in piezoelectric energy conversion is the interface circuit used between the piezoelectric transducer and storage unit. The most basic interface circuit used in this context is the circuit structure shown in Figure 1, known as the standard energy harvesting circuit [31].

The standard energy harvesting circuit has low efficiency; therefore, other circuit topologies have been developed that are shown in Figure 2 [32]. Furthermore, the principal energy harvesting circuits are compared in Table 1.

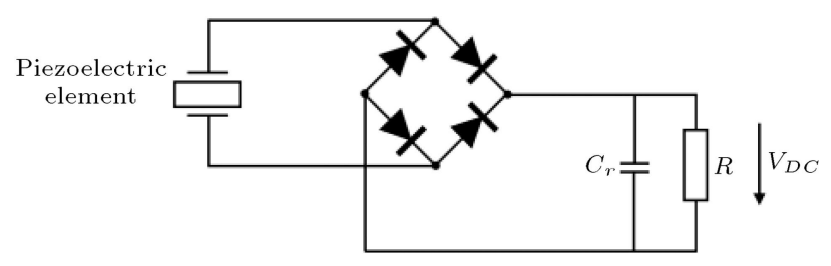

Figure 1. Standard energy harvesting circuit. 


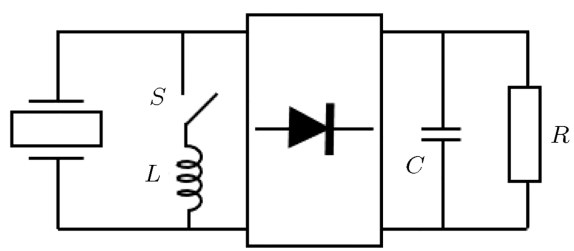

(a)

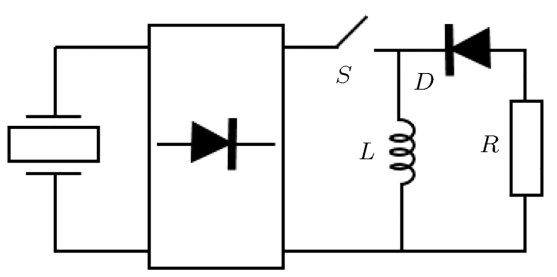

(c)

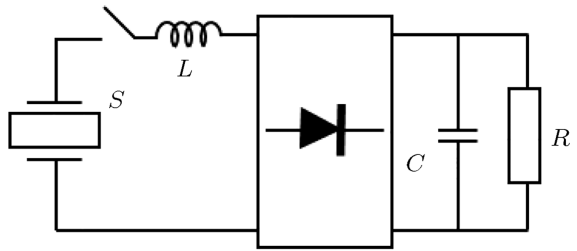

(b)

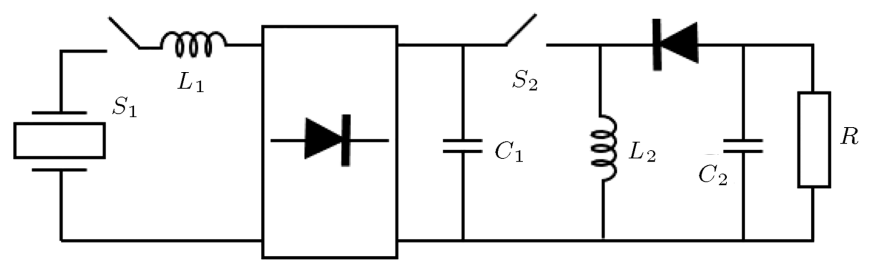

(e)

Figure 2. The principal energy harvesting circuits: (a) Parallel synchronized Switch Harvesting on Inductor (p-SSHI), (b) Series Synchronized Switch Harvesting on Inductor (s-SSHI), (c) Synchronous Electric Charge Extraction (SECE), (d) Optimized Synchronous Electric Charge Extraction (OSECE), and (e) Double Synchronized Switch Harvesting (DSSH). The principal energy harvesting circuits are compared in Table 1 [32].

Table 1. Comparison of the principal energy harvesting circuits.

\begin{tabular}{lll}
\hline Circuit topologies & \multicolumn{1}{c}{ Advantage } & \multicolumn{1}{c}{ Disadvantage } \\
\hline Standard & Simple & Load matching, power output is low \\
p-SSHI & High power output switch strategy & Load matching, inductor, switch device \\
s-SSHI & High power output switch strategy & Load matching, inductor, switch device \\
SECE & Load independents & Inductor, switch device, switch strategy \\
OSECE & Wide bandwidth switch strategy & 3-port transformer, complex circuitry \\
DSSH & Load independent & Complex circuitry, complex switch strategy \\
\hline
\end{tabular}

However, each of these energy harvesting circuits still continues to have low efficiency; therefore, a new circuit topology that can significantly increase the efficiency of a PEH is employed in this study. In the available studies, PZTs are connected directly in a series or parallel manner. The amount of energy generated depending on the connection type used in the present study is higher than that of other connected types. More details of the topology are given in Subsection 2.2 [33].

\section{Design}

\subsection{Piezoelectric effect and the sensor used}

When mechanical pressure is applied to certain crystalline materials, the electric field within the structure and, consequently, the electric potential across the structure of this material may change. The mentioned amount depends on the change in polarization density within the material. These types of crystal generate a voltage when subjected to pressure. Piezoelectric materials also exhibit an inverse effect and stresses and strains are formed when a material is exposed to an electric field [34]. The electrical and mechanical behavior can be represented by the following two equations [35]:

$$
\begin{aligned}
& S=s^{E} T+d_{t} E, \\
& D=d_{t} T+\varepsilon^{T} E,
\end{aligned}
$$

where $S$ is the mechanical strain, $E$ the electric field, $T$ the applied mechanical stress, $D$ the electric displacement, $s^{E}$ the matrix of elasticity under conditions 


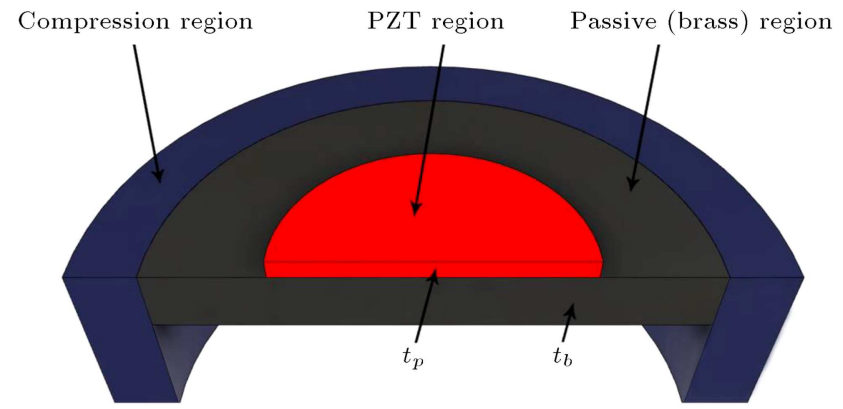

Figure 3. Structure of the unimorph diaphragm piezoelectric transducer.

of constant electric field, $\varepsilon^{T}$ the permittivity matrix at constant mechanical strain, and $d_{t}$ the piezoelectric coefficient matrix.

The piezoelectric behavior of a material is expressed by its piezoelectric coefficient, which is designated as $d_{i j}$. The first subscript, $i$, refers to the direction of the electric field resulting from the potential difference or the electric charge generated by the resulting potential difference. The second subscript, $j$, refers to the direction of the applied mechanical stress or resulting stress. Eq. (3) gives direct piezoelectric effect:

$$
d_{i j}=\left(\frac{\partial D_{i}}{\partial T_{j}}\right) .
$$

Eq. (4) gives inverse piezoelectric effect:

$$
d_{i j}=\left(\frac{\partial S_{i}}{\partial E_{j}}\right) .
$$

The piezoelectric coefficient $d_{33}$, which is related to the electric field and is obtained when strain is applied orthogonally to the polarization axis, is known to be greater than the coefficient $d_{31}$. Therefore, most of energy harvester designs rely on kinetic energy that applies strain to the material in such a way that the mechanical energy is converted through the coefficient $d_{33}$. The piezoelectric transducer in this study is also designed to operate as $d_{33}$ by applying the pressure perpendicular to the ground. The configuration of the unimorph diaphragm piezoelectric transducer used for generating electrical energy from mechanical vibration in this study is shown in Figure 3.

The piezoelectric transducer consists of two layers: the PZT with thickness of $t_{p}$ and brass with thickness of $t_{b}$, as shown in Figure 3 .

\subsection{The interface circuit used}

Piezoelectric transducers produce an alternating output voltage that must be rectified. Bridge rectifiers are widely used (as shown in Figure 1); however, two diodes carry the current in each half cycle, resulting in double voltage loss due to the diodes. Furthermore, circuit topologies in the literature do not offer a solution for the electrical connection of multiple PZTs. Therefore, this study employs a new circuit topology that connects multiple PZTs with minimum voltage loss, as shown in Figure 4 [33].

When Kirchhoff's voltage law is applied to the circuit given in Figure 4, it is observed that the output voltage is the sum of the capacitor voltages ( $V_{\text {OUT }}=$ $V_{1}+V_{2}+V_{3}+V_{4}+V_{5}+V_{6}$ ), and the voltage produced by the PZTs is rectified with minimum voltage loss since there is only a single diode in the rectification path in each half cycle. The circuit enjoys other advantages; to be specific, it prevents direct current flow through the transducers and is tolerant to failure of one or more converters. A piezoelectric vibration harvest card was designed using this circuit topology. Figure 5 shows the printed circuit board and the three-dimensional shape of the built DC harvesting card. Note that Schottky diodes are used to minimize voltage loss, and the capacitor value of $22 \mu \mathrm{F}$ is sufficiently large enough to remove AC fluctuations, yet it is sufficiently small so that the output voltage can rise rapidly to its final value.

One dc harvesting car was used for each PZT. These cards have two condensers and two diodes.

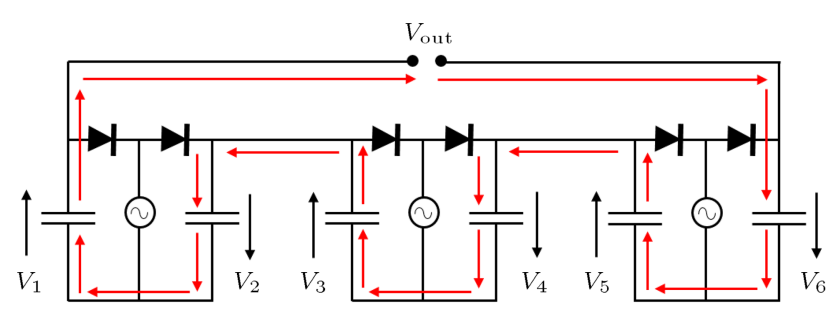

Figure 4. Circuit for analysis of the proposed topology.

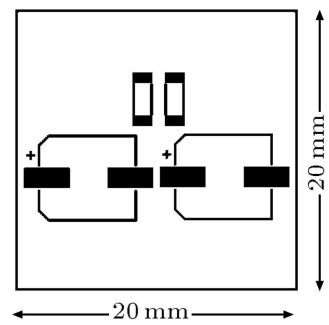

(a)

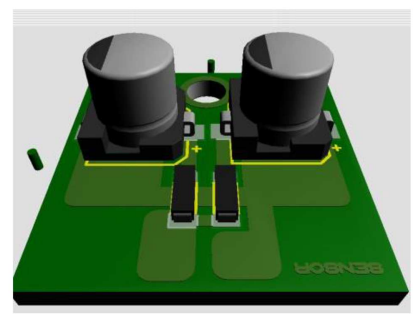

(b)

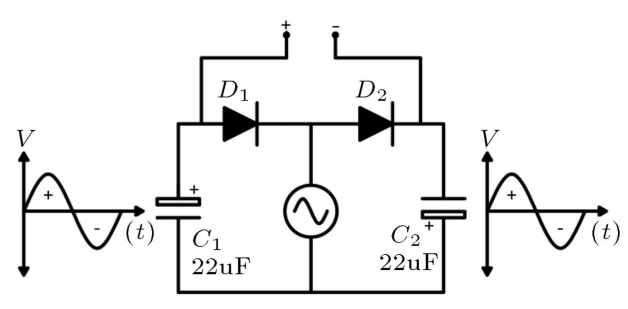

(c)

Figure 5. (a) Sensor circuit paths. (b) DC harvest card 3D view. (c) Rectifier circuit. 
Figure 5(c) shows a detailed figure of the rectifier circuit given in Figures 4 and 5 .

During the negative half cycle of the supply, $D_{1}$ diode will conduct. As a result, the current flowing through the circuit will charge the condenser $C_{1}$. In addition, there will be a voltage loss in case of voltage drop on diode $D_{1}$. Similarly, during the positive half cycle of the supply, only $D_{2}$ diode will conduct and the current flowing through the circuit will charge the condenser $C_{2}$. There will be a voltage loss due to the voltage drop on diode $D_{2}$. Since two diodes are conducting all the time in both positive and negative cycles, in a full-frame bridge rectifier, the resulting voltage loss is two times higher. Since the voltage loss is lower in this circuit, the yield will be higher [33].

In the circuit design, leg components and surface touch components were tested. As a result of the trials, surface touch was preferred to reduce the resistance caused by the leg components to zero.

\subsubsection{Electronic circuit elements used in circuit design}

- MBR0560 0.5 A 60V Schottky Diode: The main reason for selecting a Schottky diode with a SOD123 surface touch sheath structure is that it is transmitted starting from $0.45 \mathrm{~V}$ because the smaller voltage values the selected diode transmits, the less the voltage loss will be. The maximum voltage value of $60 \mathrm{~V}$ was chosen because it could carry the voltage coming from the piezoelectric sensor without loss;

- 22uF 16V SMD Electrolytic Capacitor: Electrolytic capacitor with ELEC-6.3 sheath structure can charge up to $16 \mathrm{~V}$. $22 \mathrm{uF}$ (microfarad) was chosen mainly because the frequency of the energy coming from the piezoelectric material is quite high with vibration; in other words, the capacity is kept low due to rapid transition from positive to negative. Hence, it is ensured that the transition from alternative voltage to correct voltage is complete and complementary.

\subsection{Experimental low-power generator}

The experimental generator (Figure 6) contains 20 diaphragm-type PZTs with a mass-spring structure placed on each PZT to generate the vibration from the wind. The vibration on each PZT is independent of others, and the output voltage produced by each PZT is combined using the interface circuit presented in Figure 4.

Standard PZT harvesting circuit includes a rectifier and a storing capacitor. For both positive and negative waves of piezoelectric voltage, two diodes in the bridge rectifier conduct. However, in the circuit topology used, the voltage loss is reduced by half since the piezoelectric voltage rectifies both the positive and negative waves through only one diode. Therefore, a

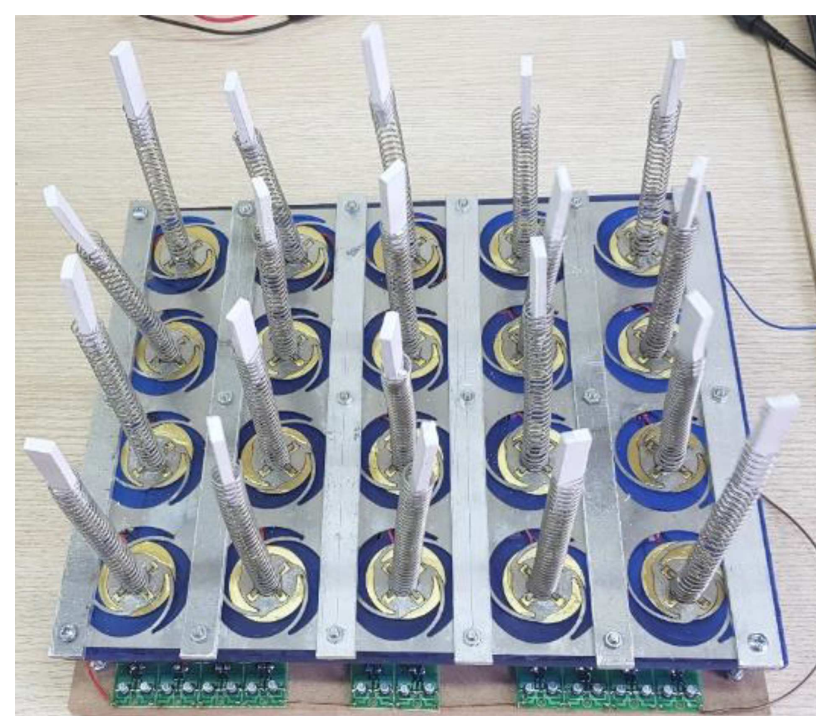

Figure 6. Experimental low-power generator.

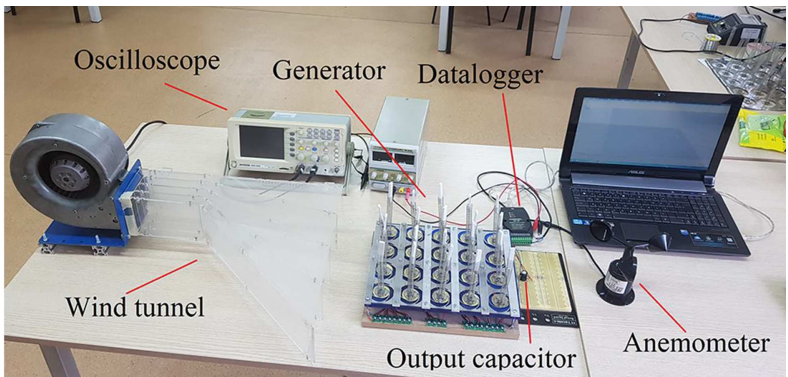

Figure 7. Experimental setup.

more efficient energy harvest can be conducted through the used circuit. In addition, the used circuit topology allows a great number of PZTs to be electrically connected to each other in an efficient way. Any one of these transducers, either short or open circuit, would not be an obstacle to the operation of the harvester.

\section{Experiments}

\subsection{Experimental setup}

The experimental setup is illustrated in Figure 7. The presented PEH was tested at nominal wind speeds of $3 \mathrm{~m} / \mathrm{s}, 6 \mathrm{~m} / \mathrm{s}, 9 \mathrm{~m} / \mathrm{s}$, and $12 \mathrm{~m} / \mathrm{s}$ using the test apparatus, as shown in Figure 7. An anemometer was also used in order to measure the wind speed. Output voltage and wind speed values were then collected using a datalogger. To generate wind at different speeds, a wind tunnel was employed. The output voltage of the generator was stored to an output capacitor of $220 \mu \mathrm{F}$.

\subsection{Experimental results}

All experiments were carried out at a certain wind speed. The collected data are given in Figures 8-11.

The output voltage at a nominal wind speed of $6 \mathrm{~m} / \mathrm{s}$ is shown in Figure 9. The output voltage at a 


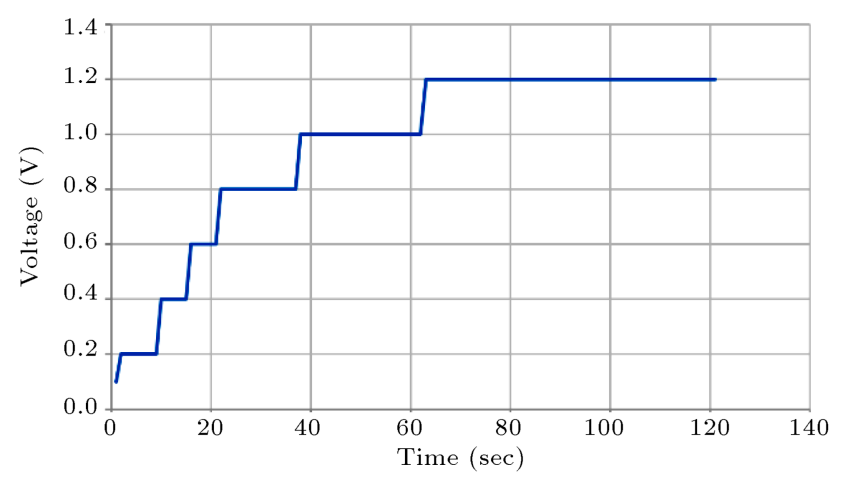

Figure 8. Output voltage for the nominal wind speed of $3 \mathrm{~m} / \mathrm{s}$.

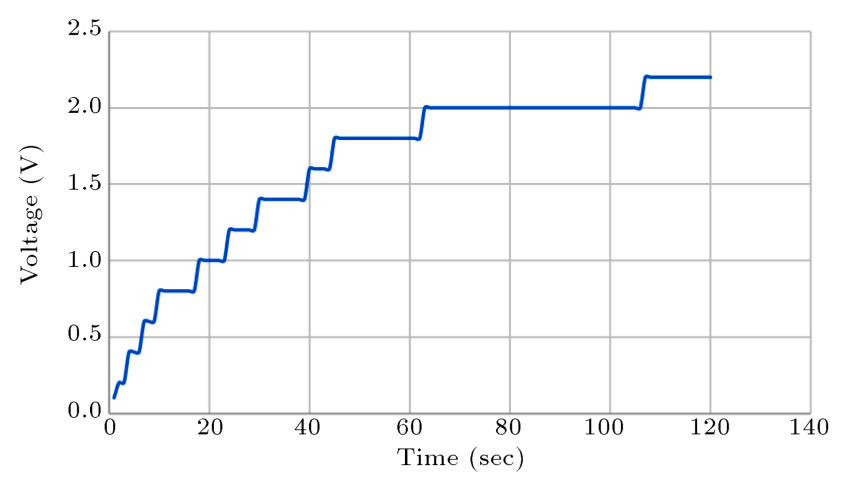

Figure 9. Output voltage for the nominal wind speed of $6 \mathrm{~m} / \mathrm{s}$.

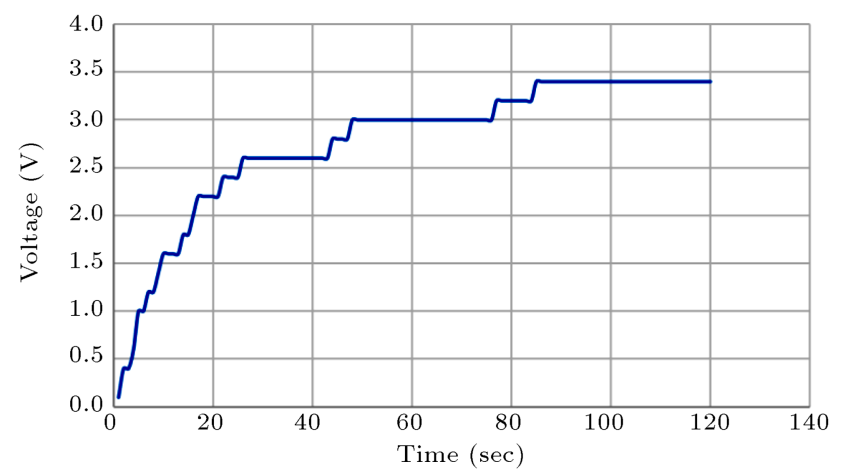

Figure 10. Output voltage for the nominal wind speed of $9 \mathrm{~m} / \mathrm{s}$.

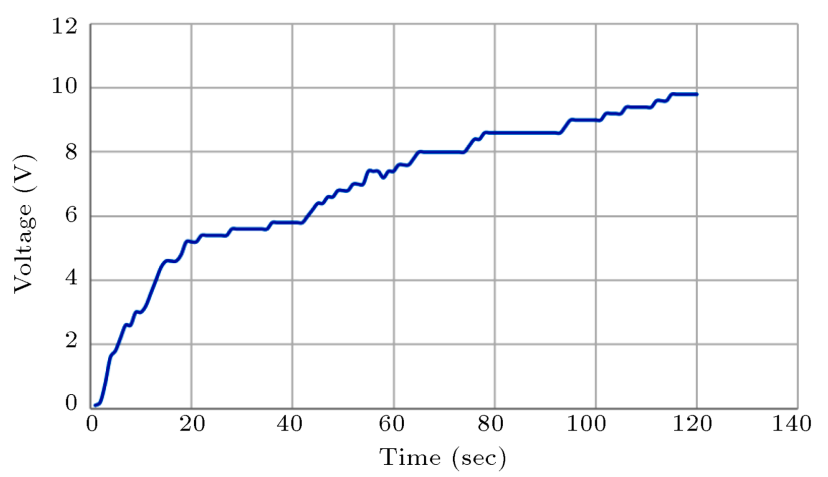

Figure 11. Output voltage for the nominal wind speed of $12 \mathrm{~m} / \mathrm{s}$.

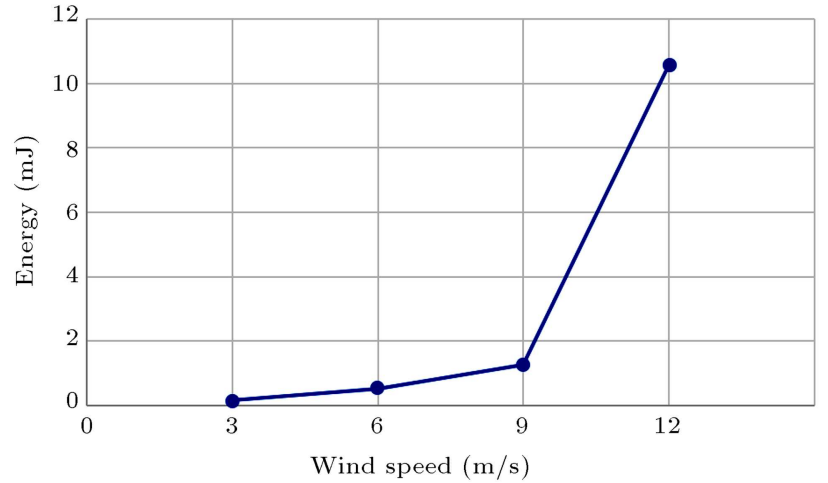

Figure 12. Energy amount in the storage capacitor versus wind speed.

nominal wind speed of $9 \mathrm{~m} / \mathrm{s}$ is shown in Figure 10 . The output voltage at a nominal wind speed of $12 \mathrm{~m} / \mathrm{s}$ is shown in Figure 11. The maximum output power values obtained for each wind speed range are given in Figure 12 .

The experimental generator was tested at nominal wind speeds of $3 \mathrm{~m} / \mathrm{s}, 6 \mathrm{~m} / \mathrm{s}, 9 \mathrm{~m} / \mathrm{s}$, and $12 \mathrm{~m} / \mathrm{s}$. The results of output voltage at these wind speeds are shown in Figures 8-12. As observed in the graph in Figure 8, the maximum output voltage obtained at a wind speed of $3 \mathrm{~m} / \mathrm{s}$ is $1.2 \mathrm{~V}$. In Figure 9, the maximum output voltage obtained at a wind speed of $6 \mathrm{~m} / \mathrm{s}$ is $2.2 \mathrm{~V}$. In the graph in Figure 10, the maximum output voltage obtained at a wind speed of $9 \mathrm{~m} / \mathrm{s}$ is $3.4 \mathrm{~V}$. In the graph in Figure 11, the maximum output voltage obtained at a wind speed of $12 \mathrm{~m} / \mathrm{s}$ is $9.8 \mathrm{~V}$. While the output voltages at wind speeds of $3 \mathrm{~m} / \mathrm{s}, 6 \mathrm{~m} / \mathrm{s}$, and $9 \mathrm{~m} / \mathrm{s}$ increased in values close to each other, the voltage value measured at a wind speed of $12 \mathrm{~m} / \mathrm{s}$ increased to approximately 8 times the first measured voltage, indicating that after a specific wind speed, these springs can swing much faster in a short span of time. Figure 12 clearly illustrates this change.

As observed in Figure 12, there is an exponential correlation between the generated energy of PZTH and wind speed because higher wind speed values cause greater vibration. However, as observed, the experimental generator has a limit value of the wind speed, which is $12 \mathrm{~m} / \mathrm{s}$. Experiments have revealed that the wind speed higher than $12 \mathrm{~m} / \mathrm{s}$ creates vibrations that may cause damage to the experimental generator. In the case of using experimental generator in higher wind speed rates, shorter springs should be selected. Experimental generators have been designed for the wind speeds of $3-12 \mathrm{~m} / \mathrm{s}$ because the selected wind speed scale is suitable for real-world applications.

\section{Conclusion}

This study presented an experimental generator based on piezoelectric energy conversion for low-power appli- 
cations. The proposed generator works on piezoelectric energy conversion. The maximum output energy values obtained in the experiments for four different wind speed ranges were $0.1 \mathrm{~mJ}, 0.8 \mathrm{~mJ}, 1.5 \mathrm{~mJ}$, and $10.8 \mathrm{~mJ}$, respectively. In the speed range of $3-9 \mathrm{~m} / \mathrm{s}$, a linear change between the wind speed and output energy was observed; however, at the nominal wind speed of $12 \mathrm{~m} / \mathrm{s}$, the output energy increased significantly, i.e., by approximately 7.3 times. This indicated that the design of the structural elements of the mass-spring system (spring constant, mass, spring length, etc.) could be improved in terms of greater efficiency.

This study employed a new circuit topology that connected multiple PZTs with minimum voltage loss. In the used circuit topology, the voltage loss was reduced by half since the piezoelectric voltage was directed both by positive and negative waves through only one diode. As a result, the efficiency of the generator was higher due to lower voltage loss.

The experimental generator utilized 20 diaphragm-type PZTs with a spring of $15 \mathrm{~cm}$ mounted at the center of each transducer that created vibrations directly on the transducer. The interface circuit provided a linear ratio between the number of PZTs and output power. For this reason, the generator structure introduced by increasing the number of PZTs was used as a battery-free power supply for devices with $\mathrm{mW}$ scale output power. In other words, it was used as a battery-free power supply for a wireless sensor node designed for forest fire detection as it was mounted on the tree trunk. Furthermore, the introduced test apparatus can be used as a test apparatus for undergraduate and postgraduate studies in the field of piezoelectric energy conversion.

\section{References}

1. Chin-Lung, Y., Kuan-Wei, C., and Chung-De, C. "Model and characterization of a press-button-type piezoelectric energy harvester", IEEE/ASME Transactions on Mechatronics, 24(1), pp. 132-143 (2019).

2. Schoeftner, J. and Buchberger, G. "A contribution on the optimal design of a vibrating cantilever in a power harvesting application-optimization of piezoelectric layer distributions in combination with advanced harvesting circuits", Eng Struct., 53, pp. 92101 (2013).

3. Luo, Q. and Tong, L. "Design and testing for shape control of piezoelectric structures using topology optimization", Eng. Struct., 97, pp. 90-104 (2015).

4. Hongseok, L., Hongseok, J., Jongkyu, P., et al. "Design of a piezoelectric energy-harvesting shock absorber system for a vehicle", Integrated Ferroelectrics, 141, pp. 32-44 (2013).

5. Wei, C. and Taghavifar, H. "A novel approach to energy harvesting from vehicle suspension system: half-vehicle model", Energy, 134, pp. 279-288 (2017).
6. Khoshnoud, F., Sundar, D.B., Badi, M.N.M., et al. "Energy harvesting from suspension systems using regenerative force actuators", Int. J. Veh. Noise Vib., 9(3-4), pp. 294-311 (2013).

7. Takashi, O. and Kanae, H. "Bioinspired flappingwing robot with direct-driven piezoelectric actuation and its takeoff demonstration", IEEE Robotics and Automation Letters, 3(4), pp. 4217-4224 (2018).

8. Xie, X.D., Wang, Q., and Wu, N. "Energy harvesting from transverse ocean waves by a piezoelectric plate", International Journal of Engineering Science, 81, pp. 41-48 (2014).

9. Song, R., Shan, X., Lv, F., et al. "A study of vortexinduced energy harvesting from water using PZT piezoelectric cantilever with cylindrical extension", Ceramics International, 41, pp. 768-773. (2015).

10. Shan, X., Song, R., Liu, B., et al. "Novel energy harvesting: A macro fiber composite piezoelectric energy harvester in the water vortex", Ceramics International, 41, pp. 763-767 (2015).

11. Na, Y., Lee, H.S., and Park, J.K. "A study on piezoelectric energy harvester using kinetic energy of ocean", Journal of Mechanical Science and Technology, 32(10), pp. 4747-4755 (2018).

12. Acciari, G., Caruso, M., Miceli, R., et al. "Piezoelectric rainfall energy harvester performance by an advanced Arduino-based measuring system", IEEE Transactions on Industry Applications, 54(1), pp. 458-468 (2018).

13. Wang, W., Cao, J., Bowen, C.R., et al. "Optimum resistance analysis and experimental verification of nonlinear piezoelectric energy harvesting from human motion", Energy, 118, pp. 221-230 (2017).

14. Türkmen, A.C. and Çelik, C. "Energy harvesting with the piezoelectric material integrated shoe", Energy, 150, pp. 556-564 (2018).

15. Amini, Y., Emdad, H., and Farid, M. "Piezoelectric energy harvesting from vertical piezoelectric beams in the horizontal fluid flow", Scientia Iranica, B, 24(5), pp. 2396-2405 (2017).

16. Abdelmoula, H. and Abdelkefi, A. "The potential of electrical impedance on the performance of galloping systems for energy harvesting and control applications", Journal Sound and Vibration, 370, pp. 191-208 (2016).

17. Javed, U., Dai, H.L., and Abdelkefi, A. "Nonlinear dynamics and comparative analysis of hybrid piezoelectric-inductive energy harvesters subjected to galloping vibrations", The European Physical Journal Special Topics, 224, pp. 2929-2948 (2015).

18. Yan, Z., Abdelkefi, A., and Hajj, M.R. "Piezoelectric energy harvesting from hybrid vibrations", Smart $M a$ terials and Structures, 23(2), pp. 1-14 (2014).

19. Abdelkefi, A., Hasanyan, A., Montgomery, J., et al. "Incident flow effects on the performance of piezoelectric energy harvesters from galloping vibrations", Theor. Appl. Mech. Lett., 4, 022002 (2014). 
20. Shan, X., Song, R., Fan, M., et al. "Energy-harvesting performances of two tandem piezoelectric energy harvesters with cylinders in water", Applied Science, 6(8), p. $230(2016)$.

21. Song, R., Shan, X., Lv, F., et al. "A novel piezoelectric energy harvester using the macro fiber composite cantilever with a bicylinder in water", Applied Sciences, 5(4), pp. 1942-1954 (2015).

22. Zhang, M., Liu, Y., and Cao, Z. "Modeling of piezoelectric energy harvesting from freely oscillating cylinders in water flow", Mathematical Problems in Engineering, 1, pp. 1-13 (2014).

23. McCarthy, J.M., Watkins, S., Deivasigamani, A., et al. "An investigation of fluttering piezoelectric energy harvesters in off-axis and turbulent flows", J. Wind Eng. Ind. Aerodyn., 136, pp. 101-113 (2015).

24. Zhou, S. and Wang, J. "Dual serial vortex-induced energy harvesting system for enhanced energy harvesting", AIP Advances, 8(7), p. 075221 (2018).

25. Johar, M.A., Kang, J.H., Hassan, M.A., et al. "A scalable, flexible and transparent $\mathrm{GaN}$ based heterojunction piezoelectric nanogenerator for bending, airflow and vibration energy harvesting", Applied Energy, 222, pp. 781-789 (2018).

26. Ju, S. and Ji, C.H. "Impact-based piezoelectric vibration energy harvester", Applied Energy, 214, pp. 139151 (2018).

27. Sang, Y., Huang, X., Liu, H., et al. "Vibration-based hybrid energy harvester for wireless sensor systems", IEEE Transactions on Magnetics, 48(11), pp. 44954498 (2012).

28. Akkaya Oy, S. and Özdemir, A.E. "Piezoelectric based low power wind generator design and testing", Arabian Journal for Science and Engineering, 43(6), pp. 27592767 (2018).
29. Xie, X.D. and Wang, Q. "A study on a high efficient cylinder composite piezoelectric energy harvester", Composite Structures, 161, pp. 237-245 (2017).

30. Song, H.C., Kumar, P., Maurya, D., et al. "Ultra-low resonant piezoelectric MEMS energy harvester with high power density", Journal of Micro Electromechanical Systems, 26(6), pp. 1226-1234 (2017).

31. Do, X., Nguyen, H., Han, S., et al. "Self-powered high-efficiency rectifier with automatic resetting of transducer capacitance in piezoelectric energy harvesting systems", IEEE Transactions on Very Large Scale Integration (VLSI) Systems, 23(3), pp. 444-453 (2015).

32. Li, Y. "Simple techniques for piezoelectric energy harvesting optimization", PhD Thesis, INSA de Lyon, France (2014).

33. Ozdemir, A. "A Novel circuit topology for piezoelectric transducers in a piezoelectric energy harvester", IET Renewable Power Generation, 13, pp. 2105-2110 (2019).

34. Ikeda, T., Fundamentals of Piezoelectricity, Oxford University Press, New York (1996).

35. Saida, M., Zaibi, G., Samet, M., et al. "Design and study of piezoelectric energy harvesting cantilever from human body", Hammamet, Tunisia, Mar. 19-22, pp. 164-168 (2018).

\section{Biography}

Sibel Akkaya Oy received her BSc degree in Electrical Education from Dicle University, Turkey in 2005 and MSc degree in Electrical Education from Firat University, Turkey in 2007. She received her PhD degree in Electrical Education from Gazi University, Turkey in 2014. Her research focuses on the electrical machine design and renewable energy. 